\title{
Rates of Staphylococcus Aureus and Methicillin-resistant Staphylococcus Aureus Nasal Carriage Infections among Nursing Students
}

\author{
Og Son Kim ${ }^{1}$, JongEun Yim $^{2}$ and MiYang Jeon ${ }^{3 *}$ \\ ${ }^{1}$ Department of Nursing Science, Sangji University, Wonju, Korea \\ ${ }^{2}$ Department of Physical Therapy, Sahmyook University, Seoul, Republic of Korea \\ ${ }^{3}$ Department of Nursing Institute of Health Science, Gyeongsang National \\ University, Jinju, Korea \\ myjeon68@hanmail.net
}

\begin{abstract}
Staphylococcus aureus is considered one of the most frequently occurring communityand hospi-tal-associated pathogens. Infectious diseases caused by Staphylococcus aureus are in various forms, ranging from mild skin infection, endocarditis, to fulminant septicemia. The purpose of this study was to determine the prevalence of methicillinresistant Staphylococcus aureus (MRSA) nasal carriage rates among nursing students. The relevant data were collected using a questionnaire, and microbial samples were collected from the nasal cavities of 215 nursing students. From the collected specimens, strains of Staphylococcus aureus were isolated and subjected to antibiotic susceptibility tests. Of 215 students, 62 (28.8\%) were nasal carriers of Staphylococcus aureus, and three students (1.4\%) were nasal carriers of MRSA. None of the three students with MRSA had any history of hospitalization over the past 12 months and had not provided nursing care for MRSA patients. However, two students among these three had received antibiotics. All MRSA isolated from these three students were mupirocin-sensitive. According to the results, the rates of MRSA nasal carriage among the nursing students were lower than those of patients or other healthcare workers. Thus, routine screening for nasal MRSA culturing is not recommended in students who practice in the clinic. However, students should be educated about infection control in order to prevent practice-related MRSA nasal.
\end{abstract}

Keywords: Infection, Nasal carriage rates, Nursing student, Methicillin-resistant Staphylococcus aureus, Staphylococcus aureus

\section{Introduction}

Despite advances in medicine, including widespread adoption of sterilized products, hygienic medical environments, disinfection, sterilization, and antibacterial materials, medicine-related infections continue to occur due to various invasive treatments and use of immunosuppressive drugs [1]. Medicine-related infections not only raise death rates, but also increase hospitalization periods leading to discomfort in patients in terms of expenses and infections; therefore, efforts are being made to prevent medicine-related infections [2]. In Korea, the Korean Society for Nosocomial Infection Control and the Center for Disease Control has been operating the Korean Nosocomial Infections Surveillance System (KONIS) since 2006; the program aims to identify and control domestic medicinerelated infections [3].

A number of pathogenic bacteria cause medicine-related infections, but the most commonly reported is Staphylococcus aureus [3]. Based on its resistance to 
methicillin antibiotics, Staphylococcus aureus may be categorized as methicillinsusceptible Staphylococcus aureus (MSSA) and methicillin-resistant Staphylococcus aureus (MRSA); medicine-related infections are mostly caused by MRSA. In an investigation involving all intensive care units in Korea in 2008, under the national hospital infection surveillance system, the single most common bacterium that caused medicine-related infection was Staphylococcus aureus at $21.1 \%$, and among antibiotic-resistant strains of Staphylococcus aureus, MRSA was reported at $89.7 \%$ [3]. MRSA, which is a major cause of hospital infections, is commonly found in the nasal cavity and can cause resident flora infection. Furthermore, medicine-related infections due to MRSA occur due to opportunistic infections originating from infected medical staff, hospital environment, other infected patients, or the patient's own resident flora $[3,4]$.

During an MRSA outbreak, MRSA nasal carriage rate increases, and it has been reported that by treating carriers, medicine-related infection due to MRSA can be reduced. Thus, nasal carriage of MRSA in medical staff or patients may play an important role in causing medicine-related infections. Consequently, in order to prevent medicine-related infections caused by MRSA in medical institutes, various infection control measures are being conducted including screening and treatment of carriers $[5,6]$.

Recently, MRSA nasal carriage rates are being reported for patients in hospitals, and for people in the community[7,8]. There is a growing interest in ascertaining the adult MRSA nasal carriage rates in the community, because MRSA that was acquired during a hospital visit can spread through the nasal carriers in the communities where they reside. According to a domestic study, of 179 MRSA strains acquired from samples collected from patients in wards and intensive care units, six strains (3.4\%) were unrelated to the hospital, and were possibly acquired from the community [9]. In addition to hospitalized patients, MRSA-infected medical staff may facilitate the spread of MRSA to other patients; therefore, appropriate management is necessary. As the Korean medical system does not screen for MRSA at the time of hospitalization, local MRSA nasal carriage rates can have a critical impact, particularly on patients with weak immune systems.

Because nursing students are not officially medical staff, they are not subjected to routine MRSA inspections. However, during field work at the hospital, nursing students are directly involved with patient care and treatment; in case nursing students are infected with MRSA, there is a risk of spreading it to patients. Nursing students are also at higher risk of being exposed to MRSA from the patients or the environment. Therefore, it is necessary to systematically manage MRSA exposure through a surveillance system, before and after field work at the hospital.

In a study of MRSA nasal carriage rates among nursing students, pharmaceutical students, dental students, and medical students in Iraq, the rates of MRSA nasal carriage were much higher rates for nursing students, at $14.3 \%, 2.1 \%, 2.2 \%$, and $2.2 \%$ in nursing, pharmaceutical, dental, and medical students, respectively[10]. Nursing students encounter more patients than other health and medical personnel; therefore, an MRSA infection management system is essential for nursing students.

Since there are no studies on MRSA nasal carriage rates on trainees in hospitals, the MRSA nasal carriage rate of students doing field work in hospitals are unknown. However, because nursing students have prolonged contact with patients during field work, they have greater exposure to MRSA infections, and are more likely to spread MRSA to patients. Hence, it is necessary to create a hospital infection management system for nursing students. Therefore, by identifying the rates of Staphylococcus aureus and MRSA nasal carriage in nursing students, this study aimed to evaluate the possibility of spreading MRSA by nursing students, and creating an effective system for prevention, education, and management of infection. 


\section{Methods}

\subsection{Participants}

Convenience sampling was conducted on nursing students enrolled at $\mathrm{K}$ University in Chungcheongbuk-do from April 18 to May 30, 2011. Specimens were collected from an estimated population of 30,813 nursing students nationwide. The inclusion criterion of the participants was limited to only the nursing students who had previous experience in clinical practice. The students were either in their second or third year at three-year colleges, or were juniors and seniors at four-year colleges. While setting $95 \%$ reliability levels and an error range of $0.7 \%, 195$ subjects were needed [11]. Based on this requirement, and taking into consideration the disqualification of research subjects, data were collected from 250 students; of these, the 215 students who answered the questionnaires appropriately and participated in the microbiological examinations were chosen as the final research subjects.

\subsection{Procedure}

This study was carried out after review by the institutional review board of $\mathrm{K}$ University (IRB No. 2011-001). From April 18 to May 30, 2011, the author(s) visited the school by grade and explained to the students that personal information would not be disclosed for reasons other than for research, and that participation would be voluntary. Only those who agreed to participate in the study signed the consent form for nasal cavity examinations. Prior to distribution, specimens and their corresponding questionnaires were assigned a unique number to aid in identification. The subjects were required to sign the attached informed consent form prior to the completing the questionnaires, which were submitted to the representatives of each grade, and collected by grade.

\subsection{Outcome Measures}

2.3.1. General Parameters: The questionnaire was based on the results of preceding studies on factors pertinent to MRSA carriers. General parameters included 11 questions including gender, age, school year, clinical work period, administration of antibiotics in the past 12 months, hospitalization in the past 12 months, hand hygiene after contact with patients, nursing patients with MRSA, entry into rooms of patients with MRSA, education on MRSA infection management, and awareness of their potential of being MRSA nasal carriers. The content validity of the questionnaire was reviewed and advised by 2 infection control nurses with more than 10 years of career in infection control.

2.3.2. Microorganism Culturing Examination: Two sterilized cotton swabs were soaked in sterile distilled water and rotated $360^{\circ}$ five times inside the left and right nasal cavities to extract specimens, and then placed in Stuart's transport media [8]. After sealing the transport media, microbiological analysis was carried out at $\mathrm{E}$ institute.

At $\mathrm{E}$ institute, the specimens were cultured in an incubator $\left(37^{\circ} \mathrm{C}\right)$ for $24-48$ hours after inoculating in a blood agar plate. After culturing, the bacterial species and anti-bacterial susceptibility of the bacterial colonies were checked using the VITEK 2 system (BioMérieux, Inc, France) and AST-P601 reagent, and was analyzed according to the CLSI M100-S20 standards. 


\subsection{Statistical Analysis}

The collected data were analyzed using SPSS Windows (Version 18.0, Chicago, USA). The frequencies and percentages of infection-related parameters, including the Staphylococcus aureus and MRSA carrier rates were analyzed, and the differences between general parameters and infection-management parameters of Staphylococcus aureus and MRSA carriers and non-carriers were examined using the chi-square test and t-test. The frequencies and percentages of the status of MRSA infection awareness in nursing students were analyzed.

\section{Results}

\subsection{General and Infection-related Parameters of Subjects}

Analysis of the general parameters of research subjects revealed that, of the 215 students, $93.0 \%$ were female. The median age was $21.92 \pm 3.52,55.3 \%$ were second-year students and $44.7 \%$ were third-year students, with clinical work periods of $12.44 \pm 5.65$ weeks. Analysis of the infection-related parameters of research subjects revealed that $35.3 \%$ had been administered antibiotics in the past twelve months, while $6 \%$ had been hospitalized; $98.6 \%$ practiced hand hygiene after contact with patients, $36.7 \%$ had entered hospital rooms with MRSA patients, while $31.2 \%$ had cared for patients infected with MRSA. While $40.9 \%$ of the students had been educated on MRSA infection management, only $18.5 \%$ were aware of their potential as MRSA-carriers, and $81.5 \%$ were not aware of the possibility of being carriers (Table 1 ).

\subsection{Staphylococcus Aureus and MRSA Carrier Rates}

Of the 215 nursing students, Staphylococcus aureus was found in the nasal cavity of $28.2 \%$ (62 students), while MRSA was detected in 1.4\% (3 students).

Table 1. General Characteristics of the Subjects ( $N=215)$

\begin{tabular}{|c|c|c|c|c|}
\hline \multirow{2}{*}{\multicolumn{2}{|c|}{ Characteristics }} & \multirow{2}{*}{$\begin{array}{l}\text { Total subject } \\
\mathrm{M} \pm \mathrm{SD} / \mathrm{n}(\%)\end{array}$} & \multirow{2}{*}{\multicolumn{2}{|c|}{ Colonization n (\%) }} \\
\hline & & & & \\
\hline \multirow{3}{*}{ Gender } & & & & \\
\hline & Male & $15(7.0)$ & $5(8.5)$ & $0(0.0)$ \\
\hline & Female & $200(93.0)$ & $57(91.5)$ & $3(100.0)$ \\
\hline Age (year) & & $21.92 \pm 3.52$ & $21.97 \pm 3.48$ & $21.33 \pm 1.53$ \\
\hline \multirow{2}{*}{ Year grade } & 2 & $119(55.3)$ & $31(50.8)$ & $1(33.3)$ \\
\hline & 3 & $96(44.7)$ & $31(49.2)$ & $2(66.7)$ \\
\hline Period of clinical practice (week) & & $12.44 \pm 5.65$ & $12.68 \pm 5.16$ & $14.67 \pm 5.77$ \\
\hline \multirow{2}{*}{ Use of antibiotics in the past 12 months } & Yes & $76(35.5)$ & $20(30.5)$ & $2(66.7)$ \\
\hline & No & $139(64.7)$ & $42(69.5)$ & $1(33.3)$ \\
\hline \multirow[t]{2}{*}{ Hospitalization in the past 12 months } & Yes & $13(6.0)$ & $2(3.4)$ & $0(0.0)$ \\
\hline & No & $202(94.0)$ & $60(96.6)$ & $3(100.0)$ \\
\hline \multirow{2}{*}{$\begin{array}{l}\text { Hand hygiene after contact with a } \\
\text { patient }\end{array}$} & Yes & $212(98.6)$ & $62(100.0)$ & $3(100.0)$ \\
\hline & No & $3(1.4)$ & $0(0.0)$ & $0(0.0)$ \\
\hline \multirow{2}{*}{$\begin{array}{l}\text { Nursing experience with an MRSA } \\
\text { patient }\end{array}$} & Yes & $67(31.2)$ & $23(39.0)$ & $0(0.0)$ \\
\hline & No & $148(68.8)$ & $39(61.0)$ & $3(100.0)$ \\
\hline \multirow[t]{2}{*}{ Entered to the MRSA patient's room } & Yes & $79(36.7)$ & $28(45.8)$ & $1(33.3)$ \\
\hline & No & $136(63.3)$ & $34(54.2)$ & $2(66.7)$ \\
\hline \multirow[t]{2}{*}{ Education on MRSA } & Yes & $88(40.9)$ & $27(44.1)$ & $1(33.3)$ \\
\hline & No & $127(59.1)$ & $35(55.9)$ & $2(66.7)$ \\
\hline \multirow[t]{2}{*}{ Possibility of MRSA nasal carrier } & Yes & $40(18.5)$ & & \\
\hline & No & $175(81.5)$ & & \\
\hline
\end{tabular}

*S. aureus: Staphylococcus aureus

**MRSA: Methicillin-Resistant Staphylococcus Aureus 


\subsection{Antibiotic Susceptibility of Staphylococcus aureus}

The results of the antibiotic susceptibility tests on Staphylococcus aureus indicated that penicillin-tolerance was the highest (91.9\%), followed by fusidic acid (25.8\%), erythromycin (16.1\%), and clindamycin $(14.5 \%)$. Oxacillin, which was used as a substitute of methicillin, showed a tolerance of $4.8 \%$, and no tolerance was evident for habekacin, linezolid, mupirocin, rifampin, teicoplanin, and vancomycin.

\subsection{Differences in General and Infection-related Parameters in Staphylococcus aureus Carriers and Non-carriers}

Upon comparing the general parameters of Staphylococcus aureus carriers and non-carriers, $91.5 \%$ of carriers and $93.4 \%$ of non-carriers were female, showing no statistically significant differences $(\chi 2=0.15, p=0.699)$. The age of the carriers was $21.45 \pm 2.98$ years and non-carriers was $21.97 \pm 3.48$ years showing no significant difference $(\mathrm{t}=1.03, p=0.302)$. By grade, $50.8 \%$ of the carriers were second-year students and $49.2 \%$ were third-year students; among non-carriers, $57.5 \%$ were second-year students and $42.5 \%$ were third-year students, showing no significant difference $(\chi 2=1.01, p=0.315)$. By duration of field work, carriers spent an average of $12.49 \pm 6.33$ weeks and non-carriers spent $12.68 \pm 5.16$ weeks, and the difference in duration was not significantly different $(\mathrm{t}=-0.21, p=0.834)$.

\section{Table 2. Comparison of Characteristics in Carriers and non-carriers of Staphylococcus aureus}

\begin{tabular}{|c|c|c|c|c|c|}
\hline \multicolumn{2}{|l|}{ Characteristics } & \multirow{2}{*}{$\begin{array}{l}\text { S. aureus* } \\
\text { carriers } \\
(\mathrm{n}=62) \\
\mathrm{M} \pm \mathrm{SD} / \mathrm{n}(\%) \\
5(8.5)\end{array}$} & \multirow{2}{*}{$\begin{array}{l}\text { S. aureus } \\
\text { noncarriers } \\
(\mathrm{n}=153) \\
\mathrm{M} \pm \mathrm{SD} / \mathrm{n}(\%)\end{array}$} & \multirow{2}{*}{$\begin{array}{l}\chi^{2} / \mathrm{t} \\
0.15\end{array}$} & \multirow{2}{*}{$\begin{array}{l}\mathrm{p} \\
.699\end{array}$} \\
\hline Gender & Male & & & & \\
\hline & Female & $57(91.5)$ & 143(93.4) & & \\
\hline Age (year) & & $21.45 \pm 2.98$ & $21.97 \pm 3.48$ & 1.03 & .302 \\
\hline \multirow[t]{2}{*}{ Year grade } & 2 & $31(50.8)$ & $88(57.5)$ & 1.01 & .315 \\
\hline & 3 & $31(49.2)$ & $65(42.5)$ & & \\
\hline Period of clinical practice (week) & & $12.49 \pm 6.33$ & $12.68 \pm 5.16$ & $\overline{0} .21$ & .834 \\
\hline \multirow[t]{2}{*}{ Use of antibiotics in the past 12 months } & Yes & $20(30.5)$ & $57(37.3)$ & 0.48 & .489 \\
\hline & No & $42(69.5)$ & $96(62.7)$ & & \\
\hline \multirow[t]{2}{*}{ Hospitalization in the past 12 months } & Yes & $2(3.4)$ & $11(7.2)$ & 1.22 & .269 \\
\hline & No & $60(96.6)$ & $142(92.8)$ & & \\
\hline \multirow[t]{3}{*}{ Hand hygiene after contact with a patient } & Hand washing & $28(45.2)$ & $72(47.4)$ & 1.42 & .492 \\
\hline & Hand sanitizer & $34(54.8)$ & $77(50.7)$ & & \\
\hline & No & $0(0.0)$ & $3(2.0)$ & & \\
\hline Frequency of hand hygiene (number/day) & & $7.03 \pm 3.24$ & $6.94 \pm 3.18$ & $\begin{array}{l}- \\
0.19\end{array}$ & .849 \\
\hline \multirow[t]{2}{*}{$\begin{array}{l}\text { Nursing experience with an MRSA } \\
\text { patient }\end{array}$} & Yes & $23(39.0)$ & $44(28.8)$ & 1.43 & .232 \\
\hline & No & $39(61.0)$ & $109(71.2)$ & & \\
\hline \multirow[t]{2}{*}{ Entered a MRSA patient's room } & Yes & $28(45.8)$ & $51(33.3)$ & 2.66 & .103 \\
\hline & No & $34(54.2)$ & $102(66.7)$ & & \\
\hline \multirow[t]{2}{*}{ Education on MRSA } & Yes & $27(44.1)$ & 61(39.9) & 0.25 & .619 \\
\hline & No & $35(55.9)$ & $92(60.1)$ & & \\
\hline
\end{tabular}

\footnotetext{
*S. aureus: Staphylococcus aureus
} 
Upon comparing the infection-related parameters of Staphylococcus aureus carriers with non-carriers, the rate of antibiotic use in the past twelve months was $30.5 \%$ for carriers, and $37.3 \%$ for non-carriers, showing no significant difference $(\chi 2=0.48, p=.489)$. The rates of hospitalization in the past twelve months, were $3.4 \%$ for carriers and $7.2 \%$ for non-carriers, showing no significant difference $(\chi 2=1.22, p=0.269)$. The results of the method of hand hygiene after nursing patients indicated that $45.2 \%$ of the carriers washed their hands and $54.8 \%$ used hand disinfectants, while for non-carriers, $47.4 \%$ washed hands, $50.7 \%$ used hand disinfectants, and $2.0 \%$ did not take any hygiene action, showing no significant difference $(\chi 2=1.42, p=0.492)$. Results for the number of times hands were washed indicated that carriers washed their hands $7.03 \pm 3.24$ times and non-carriers $6.94 \pm 3.18$ times, showing no significant difference $(\mathrm{t}=-0.19, \mathrm{p}=0.849)$. Results for experience in nursing MRSA-infected patients indicated that $39.0 \%$ of carriers and $28.8 \%$ non-carriers had cared for MRSA-infected patients, showing no significant difference $(\chi 2=1.43, p=0.232) ; 45.8 \%$ of carriers and $33.3 \%$ of non-carriers had entered rooms with MRSA patients, showing no significant difference $(\chi 2=2.66, p=0.103)$, while $44.1 \%$ of carriers and $39.9 \%$ of non-carriers had been educated on MRSA infection management, showing no statistically significant difference $(\chi 2=0.25, \mathrm{p}=.619)$ (Table 2$)$.

\subsection{Differences in General and Infection-management Parameters of MRSA Carriers and Non-carriers}

When comparing the general parameters of MRSA carriers with non-carriers, $100.0 \%$ of MRSA carriers were female and $92.5 \%$ were MRSA non-carriers, but did not show statistically significant differences $(\chi 2=0.25, p=0.621)$. By age, MRSA carriers were an average $21.33 \pm 1.53$ years old while MRSA non-carriers were $21.83 \pm 3.37$ years old, showing no significant difference $(\mathrm{t}=-0.25, p=0.800)$. By grade, $33.3 \%$ of MRSA carriers were first-year students and $66.7 \%$ were third-year students, while $55.7 \%$ of MRSA non-carriers were second-year students and $44.3 \%$ were third-year students, showing no significant difference. Results of clinical work duration indicated that carriers spent an average of $14.67 \pm 5.77$ weeks while noncarriers spent $12.51 \pm 6.01$ weeks, showing no statistically significant differences $(\mathrm{t}=$ $0.62, p=0.538$ ).

Upon comparing the infection-related parameters of MRSA carriers and non-carriers, $66.7 \%$ of MRSA carriers had received antibiotics in the past twelve months, which was higher than the $35.4 \%$ of non-carriers, but there was no statistically significant difference $(\chi 2=1.26, p=0.262)$. The hospitalization rates over the past twelve months were $0.0 \%$ for carriers and $6.1 \%$ for non-carriers, showing no significant difference $(\chi 2=0.20, p=0.658)$. When comparing the hand hygiene methods after contact with patients, $33.3 \%$ of carriers washed hands and $66.7 \%$ used hand disinfectants, while $46.9 \%$ of non-carriers washed hands, $51.7 \%$ used hand disinfectants, and $1.4 \%$ did not practice hand hygiene, showing no significant difference between the two groups $(\chi 2=0.29, p=0.866)$. Results of the number of times hands were washed/day indicated that carriers washed hands $6.67 \pm 2.89$ times and non-carriers $6.97 \pm 3.20$ times, showing no significant difference $(\chi 2=-0.16$, $p=0.870$ ). Results of experience caring for MRSA patients indicated that $0.0 \%$ of carriers, and $31.6 \%$ for non-carriers cared for MRSA-infected patients; $36.8 \%$ of carriers and $35.8 \%$ for non-carriers entered rooms with MRSA patients $(\chi 2=1.26, p=0.292)$. Of the carriers, $33.3 \%$ had received MRSA infection management education, and $41.0 \%$ of noncarriers had experience, showing no statistically significant difference $(\chi 2=0.07, p=0.788)$ (Table 3).

\subsection{Perception of MRSA Infection Possibility of Nursing Students}

When asked "Do you think there is any possibility that you are infected with MRSA?," only $18.5 \%$ of nursing students replied yes. To the question, "Why do you think MRSA 
infection is possible?," $59.0 \%$ replied that it was because of contact with MRSA patients, while $10.3 \%$ mentioned field work at the hospital, and $5.1 \%$ claimed poor hand-hygiene, answers from the remaining $2.6 \%$ included lack of knowledge of the patient's infected status, contact with too many people, lack of protective equipment, improper disinfection, poor hand-hygiene, exposure to too many MRSA patients, lack of awareness about MRSA, group-accommodation facilities, inadequate hand washing, PR through public media, and due to catching colds, each. The reasons nursing students claimed no possibility of being infected with MRSA were as follows: frequent hand washing (40.1\%), no contact with MRSA patients (20.9\%), few opportunities to come into contact with MRSA patients (18.0\%), healthy (4.3\%), maintain cleanliness (3.5\%), use masks and follow infection management guidelines (2.3\%), no MRSA infection factors (1.7\%), use gloves $(1.2 \%)$, use disinfect properly $(0.6 \%)$, and low probability of infection $(0.6 \%)$.

Table 3. Comparison of Characteristics in Carriers and non-carriers of methicillin-resistant Staphylococcus aureus

\begin{tabular}{|c|c|c|c|c|c|}
\hline \multicolumn{2}{|l|}{ Characteristics } & \multirow{2}{*}{$\begin{array}{l}\text { MRSA } \\
\text { carriers } \\
(\mathrm{n}=3) \\
\mathrm{M} \pm \mathrm{SD} / \mathrm{n}(\%) \\
0(0.0)\end{array}$} & \multirow{2}{*}{$\begin{array}{l}\text { MRSA } \\
\text { noncarriers } \\
(\mathrm{n}=212) \\
\mathrm{M} \pm \mathrm{SD} / \mathrm{n}(\%) \\
16(7.5)\end{array}$} & $\chi^{2} / \mathrm{t}$ & \multirow[t]{2}{*}{$p$} \\
\hline Gender & Male & & & 0.25 & \\
\hline & Female & $3(100.0)$ & $196(92.5)$ & & \\
\hline Age (year) & & $21.33 \pm 1.53$ & $21.83 \pm 3.37$ & $\overline{0} .25$ & .800 \\
\hline \multirow[t]{2}{*}{ Year grade } & 2 & $1(33.3)$ & $118(55.7)$ & 0.60 & $.440 *$ \\
\hline & 3 & $2(66.7)$ & $94(44.3)$ & & \\
\hline Period of clinical practice (weeks) & & $14.67 \pm 5.77$ & $12.51 \pm 6.01$ & 0.62 & .538 \\
\hline \multirow[t]{2}{*}{$\begin{array}{l}\text { Use of antibiotics in the past } 12 \\
\text { months }\end{array}$} & Yes & $2(66.7)$ & $75(35.4)$ & 1.26 & $.262 *$ \\
\hline & No & $1(33.3)$ & $137(64.6)$ & & \\
\hline \multirow[t]{2}{*}{$\begin{array}{l}\text { Hospitalization in the past } 12 \\
\text { months }\end{array}$} & Yes & $0(0.0)$ & $13(6.1)$ & 0.20 & $.658 *$ \\
\hline & No & $3(100.0)$ & 199(93.9) & & \\
\hline \multirow[t]{3}{*}{$\begin{array}{l}\text { Hand hygiene after contact with a } \\
\text { patient }\end{array}$} & $\begin{array}{l}\text { Hand } \\
\text { washing }\end{array}$ & $1(33.3)$ & $100(47.1)$ & 0.29 & $.866^{*}$ \\
\hline & $\begin{array}{l}\text { Hand } \\
\text { sanitizer }\end{array}$ & $2(66.7)$ & $109(51.4)$ & & \\
\hline & No & $0(0.0)$ & $3(1.4)$ & & \\
\hline \multicolumn{2}{|l|}{ Frequency of Hand hygiene (number/day) } & $6.67 \pm 2.89$ & $6.97 \pm 3.20$ & $\begin{array}{l}- \\
0.16\end{array}$ & .870 \\
\hline \multirow[t]{2}{*}{$\begin{array}{l}\text { Nursing experience with an MRSA } \\
\text { patient }\end{array}$} & Yes & $0(0.0)$ & $67(31.6)$ & 1.38 & $.554 *$ \\
\hline & No & $3(100.0)$ & $145(68.4)$ & & \\
\hline \multirow[t]{2}{*}{ Entered a MRSA patient's room } & Yes & $1(33.3)$ & $78(36.8)$ & 1.26 & $.292 *$ \\
\hline & No & $2(66.7)$ & $134(63.2)$ & & \\
\hline \multirow[t]{2}{*}{ Education on MRSA } & Yes & $1(33.3)$ & $87(41.0)$ & 0.07 & $.788 *$ \\
\hline & No & $2(66.7)$ & $125(59.0)$ & & \\
\hline
\end{tabular}

*Fisher's Exact Test

\section{Discussion}

Staphylococcus aureus normally exists on the skin or mucous membrane and is generally susceptible to methicillin. However, some strains of Staphylococcus aureus are resistant to methicillin, giving rise to issues of anti-bacterial resistance. 
Nasal carriage rates of MRSA are slightly different depending on study subjects. In a study conducted on 5,375 hospitalized patients at a university hospital in the United States, $581(10.8 \%)$ were nasal carriers of MRSA [12]; in a study conducted in the Netherlands on 6,496 hospitalized patients from October 2005 to June 2007, only seven $(0.11 \%)$ were nasal carriers of MRSA [13]; while the results from a study on 744 long-term residents of a hospital in southern Spain indicated that, 79 (10.6\%) were MRSA nasal carriers [14]. In one large-scale study on 32,206 patients from nine European countries, nasal carrier rates ranged $0.0-2.1 \%$, in each country [15]. In a study of 482 patients with hemodialysis in seven general hospitals in Korea, 57 (11.8\%) were nasal carriers [16].

Approximately $4.6 \%$ medical workers worldwide are MRSA carriers, but this varies in each study $[17,18]$. In a study conducted on 177 medical workers in Ethiopia, 25 (14.1\%) were MRSA nasal carriers, and the carrier rates for nurses and doctors were higher than those having other occupations [19]. In a study conducted on medical workers and students training to become emergency response workers at an emergency medical center in the midwest United States, $4.5 \%$ medical workers and $5.3 \%$ students were nasal carriers of MRSA [20]. In a study on medical staff from six hospitals in Korea, 6 of $91(6.6 \%)$ were found nasal carriers; 5 of 67 (7.5\%) were nurses and 1 of $24(4.2 \%)$ were doctors [16].

In an MRSA nasal carrier study on 203 students at the University of Texas, 15 $(7.4 \%)$ were found to be carriers [21]. In Thailand, 2 of $200(1.0 \%)$ of college students were found to be carriers [14], and in a study of 95 locations in seven dental hospital environments and 61 dental school students, MRSA was found in 13 students (21\%) and eight dental hospital environments (8.4\%) [22]. In a study conducted on MRSA nasal carriers in Iraq, out of 56 nursing students, 48 pharmaceutical students, 45 dental students, and 135 medical school students, 8 (14.3\%) nursing students, 1 (2.1\%) pharmaceutical student, 1 (2.2\%) dental student, and three $(2.2 \%)$ medical school students were reported to carry MRSA in their nasal cavities [10].

The MRSA nasal carrier rate of nursing students who participated in this study was $1.4 \%$, similar to that of patients in Europe, but lower than the overall nasal carrier rates of patients and medical staff in Korean hospitals. Further, upon comparing with domestic and foreign studies on students, MRSA nasal-carriage rates were higher in Korean patients than Thai college students [14] but were lower than those in dental school students in the United States [22] and in nursing school students in Iraq [10]. While the nasal carrier rates for Korean patients are reportedly higher than those of patients of other nationalities, the nasal carrier rates were not very high for the nursing students in this study. We propose that this may be due to a number of reasons such as limited exposure of the nursing students to MRSA patients during their clinical work, or that basic infection management and prevention rules were observed, including proper hand-hygiene after contact with patients.

In the past year, it has been reported that use of antibiotics and hospitalization are risk factors for being nasal carriers of MRSA [21, 23]. In this study, two of three MRSA nasal carriers had received antibiotics in the past twelve months, while none of the three had been hospitalized during the past twelve months. This is similar to the results in patients using antibiotics but these patients cannot be compared, as they were not hospitalized. However, because of the few students with MRSA in this study, our study has low significance as comparative data, and large-scale studies are needed in the future.

When considering the effects of hospital training on MRSA nasal carrier rates, the results of a study including 42 students without clinical work experience and 52 with clinical work experience at a medical school in Louisiana, revealed that only 
three with clinical work experience had MRSA, while none of the students without clinical work experience had MRSA [24]. In a study on 66 nursing students that examined MRSA carriers before and after clinical work, no cases of MRSA were detected prior to clinical work; however, after clinical work, $6.1 \%$ of the nursing students were found to have MRSA in their hands and nasal cavities [25]. Moreover, in an MRSA nasal carrier study on 322 medical school students from Taiwan, 2.5\% of students before training and $1.9 \%$ after training were found to be nasal carriers; field work at a hospital was not found a significant risk factor for MRSA carriers according to this study [26]. Prior hospitalization in patients has reported as a risk factor for MRSA nasal carriers, but in students, risk factors differ based on whether field work was carried out at hospitals. This difference may be attributed to basic infection management, including proper hand-hygiene and use of protective equipment, as well as participation in care for MRSA-patients. Therefore, it is necessary to educate students on prevention of MRSA infection prior to assigning field work.

Upon analyzing antimicrobial susceptibility in 253 samples Staphylococcus aureus isolated from patients in 13 medical institutes in Korea, only $4.3 \%$ were susceptible to penicillin, while $100 \%$ were susceptible to vancomycin and teicoplanin. When oxacillin was analyzed as a substitute for methicillin, only $34.0 \%$ were susceptible, while $65.6 \%$ were resistant [27]. While there were slight differences in the antibacterial susceptibility of Staphylococcus aureus strains in this study, penicillin-susceptibility was low overall, while susceptibility to vancomycin and teicoplanin was $100 \%$, similar to the rates of antibacterial susceptibility reported in previous studies.

In this study, only $1.4 \%$ of the students were nasal carriers of MRSA, at a relatively lower carrier rate than patients and medical workers. However, treatment of nasal carriers is crucial for the prevention of MRSA dissemination. All three students in this study carried MRSA strains susceptible to mupirocin, which is commonly used for treating nasal carriers; thus, carriers may be treated by covering the nasal cavity with mupirocin ointment [28]. In the event that they continue to work at the hospital without proper treatment, they may spread resistant bacteria to patients, medical staff, or other students, and so appropriate treatment is necessary.

Some of the students with MRSA in the study were verified to be MRSA nasal carrier although they did not have previous experience in MRSA patient nursing. There were studies that reported detection of MRSA in the workplace of nurses and doctors, corridors, and mobile phone of medical personnel, not in the wards of MRSA patients [29, 30], thus, it is understood that MRSA can be transmitted through indirect contact if being exposed to the environment contaminated by MRSA, without direct nursing of MRSA patients. Since community-acquired MRSA infection was reported in Korea[31], there is also a possibility to acquire MRSA in a community that has nothing to do with clinical practice.

In order to assess MRSA carrier state, this study was included only the nursing students clinical practice. It is limited so it is difficult to judge whether MRSA was acquired in the actual clinical practice or in a community that has nothing to do with the practice. Such limitation should be considered for further studies.

In addition, although only a few MRSA nasal carriers were included in this study, it is necessary to develop prevention and management programs for the students against methicillin-resistant infections for such carriers. In order to prevent methicillin-resistant infections in students, management procedures used for multidrug resistant bacteria infection, including thorough hand hygiene should be included in the program. While screening of MRSA carriers by microbial cultures can be considered for medical workers, routine examinations can be ineffective in terms of cost. Moreover, as selective examinations are recommended for high-risk 
groups of carriers [5], instead of conducting microbial tests to identify carriers among nursing students, infection management programs should be developed to prevent students from contracting multi-drug resistant bacteria such as MRSA. In case students received training at a department where there was an MRSA outbreak, selective carrier examinations and treatment should be taken into consideration.

\section{Conclusion}

This study was carried out to identify the MRSA status of nasal carriers among nursing students. Of the 215 subjects, three (1.4\%) were MRSA nasal carriers. All three students with MRSA had not been hospitalized for the past twelve months and had not participated in MRSA patient nursing. However, two had been administered antibiotics. All MRSA detected in the three students was susceptible to mupirocin. The MRSA nasal carrier rates for the nursing students in this study were lower than those for general patients and medical staff; also, since the MRSA was susceptible to mupirocin, it was recommended that regular nasal carrier examinations would not be necessary for all students who engaged in hospital field work. However, in the event that the field work was conducted during a group outbreak, examination for nasal carriers should be carefully considered and judged according to the situation. In addition, thoroughly educating students on infection management prior to clinical work is important in order to prevent them from becoming nasal carriers of MRSA.

\section{References}

[1] M. H. Jeon, W. B. Park, S. R. Kim, H. K. Chun, S. H. Han, J. H. Bang, E. S. Park, S. Y. Jeong, J. S. Eom and Y. K. Kim, "Korean nosocomial infections surveillance system, intensive care unit module report", data summary from July 2010 through June 2011. Korean Journal of Nosocomial Infection Control, vol. 17, no. 1, (2012), pp. 28-39.

[2] C. A. Umscheid, M. Rajender Agarwal MD, M. Kendal Williams MD and P. J. Brennan, "Estimating the proportion of healthcare-associated infections that are reasonably preventable and the related mortality and costs", infection control and hospital epidemiology, vol. 32, no. 2, (2011), pp. 101-114.

[3] S. O. Lee, E. S. Kim, H. Y. Kim, E. S. Park, H. Y. Jin, H. K. Ki, H. J. Choi, S. Y. Jeong, S. R. Kim and S. Kim, "Korean nosocomial infections surveillance system, intensive care unit module report", Data summary from July 2007 through June 2008. Korean Journal of Nosocomial Infection Control, vol. 13, no. 2, (2008), pp. 69-82.

[4] M. Kalmeijer, H. Coertjens, P. van Nieuwland-Bollen, D. Bogaers-Hofman, G. J. de Baere, A. Stuurman, A. Van Belkum and J. W. Kluytmans, "Surgical site infections in orthopedic surgery: the effect of mupirocin nasal ointment in a double-blind, randomized, placebo-controlled study", Clinical Infectious Diseases, vol. 35, no. 4, (2002), pp. 353-358.

[5] P. S. Pottinger, "Methicillin-Resistant Staphylococcus aureus Infections", The Medical clinics of North America, vol. 97, no. 4, (2013), pp. 601-619.

[6] N. A. Parks and M. A. Croce, "Routine screening for methicillin-resistant Staphylococcus aureus", Surgical infections, vol. 13, no. 4, (2012), pp. 223-227.

[7] K. Vardakas, I. Kontopidis, I. Gkegkes, P. Rafailidis and M. Falagas, "Incidence, characteristics, and outcomes of patients with bone and joint infections due to community-associated methicillin-resistant Staphylococcus aureus: a systematic review", European journal of clinical microbiology \& infectious diseases, vol. 32, no. 6, (2013), pp. 711-721.

[8] T. Kejela and K. Bacha, "Prevalence and antibiotic susceptibility pattern of methicillin-resistant Staphylococcus aureus (MRSA) among primary school children and prisoners in Jimma Town", Southwest Ethiopia. Ann. Clin. Microbiol. Antimicrob, vol. 12, no. 11, (2013).

[9] H. M. Mun, S. D. Kim, B. C. Chun, S. O. Lee, M. N. Kim, J. J. Sim, H. R. Choi, H. J. Park, M. K. Han, and S. H. Kwak, "Community and hospital onset methicillin-resistant Staphylococcus aureus in a tertiary care teaching hospital", Korean Journal of Nosocomial Infection Control, vol. 14, no. 1, (2009), pp. 2435 .

[10] N. Yassin and A. Hassan, "Nasal carriage of methicillin-resistant/sensitive Staphylococcus aureus among students in Faculty of Medical Sciences", Duhok University. Adv Trop Med Pub Health Int, vol. 3, (2013), pp. 65-72.

[11] Y. Tack, K. Kang, J. Kim, T. Kim, H. Kim and J. Song, "Introduction to statistics for nurse", Seoul: Fornurse, (2009). 
[12] N. Parvez, C. Jinadatha, R. Fader, T. W. Huber, A. Robertson, D. Kjar and L. K. Cornelius, "Universal MRSA nasal surveillance: characterization of outcomes at a tertiary care center and implications for infection control", Southern medical journal, vol. 103, no. 11, (2010), pp. 1084-1091.

[13] L. Bode, H. Wertheim, J. Kluytmans, D. Bogaers-Hofman, C. Vandenbroucke-Grauls, R. Roosendaal, A. Troelstra, A. Box, A. Voss and A. van Belkum, "Sustained low prevalence of meticillin-resistant< i> Staphylococcus aureus $</ \mathrm{i}>$ upon admission to hospital in The Netherlands. Journal of Hospital Infection, vol. 79, no. 3, (2011), pp. 198-201.

[14] T. Kitti, K. Boonyonying and S. Sitthisak, "Prevalence of methicillin-resistant Staphylococcus aureus among university students in Thailand", Southeast Asian Journal of Tropical Medicineand Public Health, vol. 42, no. 6, (2011), pp. 1498 .

[15] C. D. den Heijer, E. M. van Bijnen, W. J. Paget, M. Pringle, H. Goossens, C. A. Bruggeman, F. G. Schellevis and E. E. Stobberingh, "Prevalence and resistance of commensal $<\mathrm{i}>$ Staphylococcus aureus $\langle/ \mathrm{i}\rangle$, including meticillin-resistant $\langle\mathrm{i}>\mathrm{S}$ aureus $\langle/ \mathrm{i}\rangle$, in nine European countries: a cross-sectional study", The Lancet infectious diseases, vol. 13, no. 5, (2013), pp. 409-415.

[16] J. Kim, S. Lee, J. Jeong, K. H. Roh, H. Lee, S. J. Jang, H. S. Lee, J. Kim, S. H. Lee and J. S. Yeom, "Nasal Colonization and Molecular Characterization of Methicillin-Resistant Staphylococcus aureus among Hemodialysis Patients in 7 Korean Hospitals", Korean Journal of Nosocomial Infection Control, vol. 18, no. 2, (2013), pp. 51-56.

[17] M. H. Kim, W. I. Lee and S. Y. Kang, "Detection of Methicillin-Resistant Staphylococcus aureus in Healthcare Workers Using Real-Time Polymerase Chain Reaction", Yonsei medical journal, vol. 54, no. 5, (2013), pp. 1282-1284.

[18] N. Safdar and E. A. Bradley, "The Risk of Infection after Nasal Colonization with $<$ i> Staphylococcus Aureus $</ i>"$, The American journal of medicine, vol. 121, no. 4, (2008), pp. 310-315.

[19] A. Gebreyesus, S. Gebre-Selassie and A. Mihert, "Nasal and hand carriage rate of methicillin resistant Staphylococcus aureus (MRSA) among health care workers in Mekelle Hospital", North Ethiopia, Ethiopian medical journal, vol. 51, no. 1, (2013), pp. 41-47.

[20] C. Miramonti, J. A. Rinkle, S. Iden, J. Lincoln, G. Huffman, E. Riddell and M. A. Kozak, "The prevalence of methicillin-resistant Staphylococcus aureus among out-of-hospital care providers and emergency medical technician students", Prehospital Emergency Care, vol. 17, no. 1, (2012), pp. 73-77.

[21] R. E. Rohde, R. Denham and A. Brannon, "Methicillin resistant Staphylococcus aureus: carriage rates and characterization of students in a Texas university", Clinical laboratory science: journal of the American Society for Medical Technology, vol. 22, no. 3, (2008), pp. 176-184.

[22] M. C. Roberts, O. O. Soge, J. A. Horst, K. A. Ly and P. Milgrom, "Methicillin-resistant< i> Staphylococcus aureus $</ \mathrm{i}>$ from dental school clinic surfaces and students", American journal of infection control, vol. 39, no. 8, (2011), pp. 628-632.

[23] H. Al-Talib, C. Y. Yean, H. Hasan, N. Nik Zuraina and M. Ravichandran, "Methicillin-resistant Staphylococcus aureus nasal carriage among patients and healthcare workers in a hospital in Kelantan", Malaysia. Polish Journal of Microbiology, vol. 62, no. 1, (2013), pp. 109-112.

[24] C. Bellows, A. Smith, J. Wheeler and L. Morici, "Nasal carriage of methicillin-resistant Staphylococcus aureus among students at a Louisiana medical university", Brazilian Journal of Infectious Diseases, vol. 17, no. 1, (2013), pp. 118-119.

[25] R. B. Akpınar, A. Celebıglu, H. Uslu and M. Hamidullah Uyanık, "An evaluation of the hand and nasal flora of Turkish nursing students after clinical practice", Journal of clinical nursing, vol. 18, no. 3, (2009), pp. 426-430.

[26] C.-S. Chen, C.-Y. Chen and Y.-C. Huang, "Nasal carriage rate and molecular epidemiology of methicillin-resistant $<\mathrm{i}>$ Staphylococcus aureus $</ \mathrm{i}>$ among medical students at a Taiwanese university", International Journal of Infectious Diseases, vol. 16, no. 11, (2012), pp. e799-e803.

[27] J. Kim, H. Kim, W. Song, H. C. Cho, K. M. Lee and E. C. Kim, "Antimicrobial resistance profiles of Staphylococcus aureus isolated in 13 Korean hospitals", The Korean Journal of Laboratory Medicine, vol. 24, no. 4, (2004), pp. 223-229.

[28] T. Coates, R. Bax and A. Coates, "Nasal decolonization of Staphylococcus aureus with mupirocin: strengths, weaknesses and future prospects", Journal of Antimicrobial Chemotherapy, (2009), p. dkp159.

[29] J. S. Kim, O. K. Kwon, W. Song, H. S. Kim, J. Y. Park, H. C. Cho, K. M. Lee and H. R. Lee, "Isolation of Healthcare-Associated Pathogens from Cellular Phones Used by Medical Personnel", Korean Journal of Nosocomial Infection Control, vol. 15, no. 1, (2010), pp. 36-40.

[30] M. C. Faires, D. L. Pearl, O. Berke, R. J. Reid-Smith and J. S. Weese, "The identification and epidemiology of meticillin-resistant Staphylococcus aureus and Clostridium difficile in patient rooms and the ward environment", BMC Infect Dis, vol. 13, (2013), pp. 342.

[31] S. Y. Lee, J. Y. Kim, J. H. Kim, S. Y. Kim, C. Park, Y. S. Park, Y. H. Seo and Y. K. Cho, "A case of primary infective endocarditis caused by community-associated methicillin-resistant Staphylococcus aureus in a healthy individual and colonization in the family", Yonsei Med J, vol. 50, no. 1, pp. 152-5. 
International Journal of Bio-Science and Bio-Technology

Vol.7, No.5 (2015) 